\title{
A Combinatorial Clock Auction for OFDMA-based Cognitive Wireless Networks
}

\author{
Timothy K. Forde \& Linda E. Doyle \\ Centre for Telecommunications Value-chain Research (CTVR), \\ Trinity College, University of Dublin, Ireland. \\ tkforde@gmail.com, ledoyle@tcd.ie
}

\begin{abstract}
This paper presents a novel application of a market-based spectrum assignment mechanism for exploitation by cognitive radio systems. Advances in DSA policy mean that regulators are moving towards a more liberalised system of spectrum rights which give more freedom to spectrum users to choose the radio and networking technologies, services and frequencies that suit their technical and economic needs. The paper describes a combinatorial clock auction mechanism that allows for the trade of spectrum rights in the context of an OFDMAbased cognitive wireless network.

While governments can use complex and cumbersome auctions to sell off large blocks of spectrum at the macro level, it will be necessary to employ cognitive wireless networks to organise the distribution of such spectrum rights at the network level. Cognitive functionality is important so that DSA-enabled wireless networks can understand the regulatory, technical and economic context within which they find themselves. The combinatorial clock auction is a mechanism that will facilitate the multilateral trade of spectrum rights among competing cognitive radios.
\end{abstract}

\section{Dynamic Spectrum Access}

There has been much debate in recent years concerning the reform and liberalisation of RF spectrum policy in an effort to move away from the innovationstifling command-and-control system [1]-[4]. We focus on addressing the challenges that will arise in facilitating and exploiting a very liberalised market-based spectrum assignment and spectrum allocation system.

The last two decades have seen slow moves towards the market-based assignment of some bands of spectrum, e.g. 2G/3G/AWS auctions [5]. However, spectrum is still largely subject to command-and-control allocation, those bands of spectrum have been harmonised for very specific technologies and/or services. Real liberalisation should see the market, i.e. those who consume spectrum, decide on the technologies and services to which it is dynamically allocated. This commoditisation of spec- trum depends, in part, on the adoption of exclusiverights, flexible-usage policies, i.e. technology and service neutral policies. However, the auction techniques that are used for the IPO-like release of spectrum licences are not suited to network implementation due to their computational complexity and mechanism design.

While the definition of such exclusive spectrum rights is complex, De Vany has introduced the concept of the Time/Area/Spectrum bundle of rights [8], [9]. Such a bundle of rights, or licence, is defined by at least three dimensions: a geographical area, a period of time and a band of frequencies within which the licencee may operate.

The application of this market-based approach can been seen on many scales, from the marco-level (government or regulator-level) sale of large swathes of spectrum to large telcos or spectrum wholesalers, right down the micro-level sale of spectrum in networks. The liberalization of the licensing regimes, which may include opening up certain licence categories for secondary trading [6], means that licences can be traded in part or in whole, i.e. they can be partitioned in space or disaggregated in frequency.

At some point it becomes both technically and economically desirable to stop disaggregating and partitioning licences in technology and service agnostic terms, i.e. sub-dividing TAS-like rights. There comes a point when it is more efficient for the spectrum rights holder to allocate the spectrum to a particular network access technology and then to trade access to the spectrum within the confines of that access technology.

In this paper, we look at issues surrounding the resale of spectrum within the confines of a given access technology - Orthogonal Frequency Division Multiple Access (OFDMA). Section II briefly discusses OFDMAbased systems. Section III describes and discusses the combinatorial clock auction while Section IV addresses the concept of a clock auction for an OFDMA-based 
network.

\section{OFMDA-BASED NETWORKS}

One of the most recent, and high-profile, reforms in spectrum regulation is that surrounding the U.S. digital television (DTV) transition which may result in two significant advances. Firstly, the transition has freed up the so-called $700 \mathrm{MHz}$ band for new services operating under more liberal service and technology rules. Secondly, the move to DTV has created a newly regulated space for opportunistic devices to co-operate with the incumbent primary TV networks on a non-interfering basis [15]. One of the firm proposals for opportunistic use of the DTV spectrum is the IEEE 802.22 [7] cognitive radioenabled wireless regional area network standard.

In general terms, a cognitive [10] network can be described as a network that is capable of knowing and understanding the context within which it finds itself and is capable of processing and acting on that knowledge as it sees fit. It could be said that cognitive networks also embody a sense of dynamic responsiveness as actions are typically taken in response to changing circumstances and changing (radio/node/network) resource availability. We extend the concept to cognition in a wireless network to the economic sphere. The network elements must take cognisance of the price of spectrum and trade it amongst themselves, using a multi-lateral decision-making system, according to both technical and economic demands.

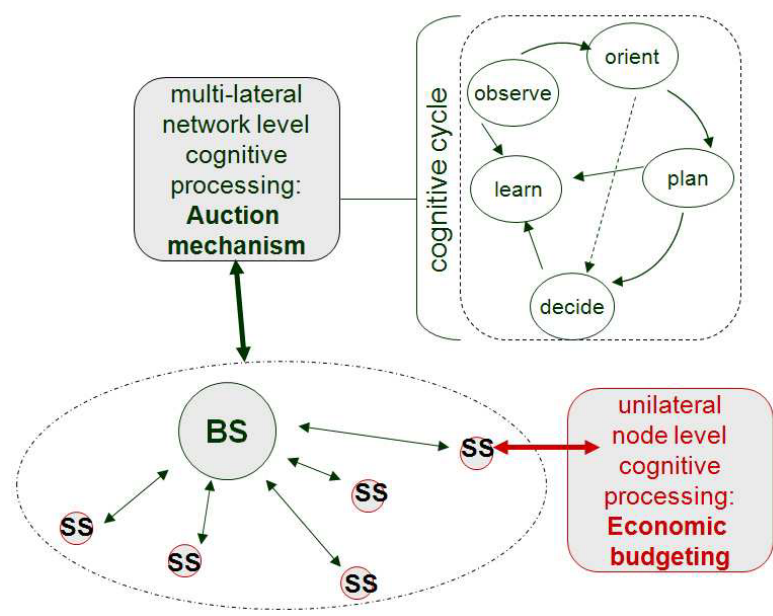

Fig. 1. An Infrastructure-based Cognitive Wireless Network

The IEEE 802.22 [7] network is a real example of a system that could be described by Figure 1. It is somewhat similar to the WiMAX (802.16) point-to-multipoint MAC. IEEE 802.22 uses a master/slave basestation (BS) to subscriber station (SS) network topology in which the
BS collates information from the SSs and learns, adapts and makes decision based on this information. All nodes can influence the collective decision through performing their own local cognitive analysis, but the decision is taken in a centralized fashion.

One of the key enablers of dynamic responsiveness to changing spectrum availability in IEEE 802.22 is its use of a scalable OFDMA radio access technology at the physical layer.

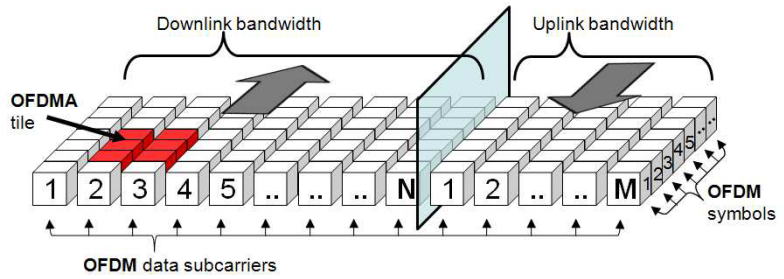

Fig. 2. A logical representation of FDD OFDMA data subcarriers and symbols

Some of the main benefits of an OFDMA physical layer are that it supports dynamic bandwidth availability, i.e. the number of subcarriers can increase or decrease as the available bandwidth changes. It also permits various options for the process of subchannelisation, i.e. the process by which OFDM sub-carriers are logically grouped together to create a channels for each user in the system. An adjacent distribution, as depicted by the OFDMA tile in Figure 2, of subcarriers allows for beam-forming and for the exploitation of multiuser diversity, i.e. when subcarriers are assigned to those users experiencing the best SNR on them. In IEEE 802.22 and mobile WiMAX (IEEE 802.16e) the MAC decides on the permutations used in the uplink and downlink according to the QoS demanded by the subscriber stations.

In this paper we move from a QoS paradigm to an exclusive access paradigm. Rather than depending on some notion of fair resource distribution, each cognitive network element is responsible for acquiring its own lease on guaranteed spectrum access rights. OFMDA, as a flexible access technique that enables the resource to be neatly carved up and packaged, represents a relatively simple example with which to elaborate the concept of spectrum trading at this level of granularity.

\section{Combinatorial Auctions}

In order for the market players to make economically efficient deals, they require a market environment that enables them to exchange messages (bids/asks) that may 
lead to a mutually acceptable bargain. The most common mechanism to enable such multi-lateral trading is some form of auction. Combinatorial auctions are very useful when there are a range of items on sale which may be logically grouped together into many different packages to suit either the buyer, the seller or both. One of the main benefits of combinatorial auctions is that they reduce the financial exposure of the traders, i.e. a buyer will either get to buy all of its target package or none of it. A broad review of combinatorial auction mechanism designs is to be found in [14].

Combinatorial auctions are rare in practice. Among the reasons for this are their complexity and the possibility that they allow for (negative) strategic bidding which can pervert the outcome of the auctions, e.g. retaliatory or jump bidding. The complexity has two main impacts: it can make the auction space too large for the bidder to figure out all the various packages that it could bid for successfully, and it can also be too complex for the auctioneer to compute the winning bidders in a reasonable time. Furthermore, the complexity of a combinatorial auction may increase as the buyers' and sellers' (the traders') expressiveness increases, i.e. as they are given increasing flexibility in types of packages of bids they are allowed to assemble. However, one of the main benefits of more expressive bidding is that it leads to a Pareto improvement in the assignment of the resource [12].

\section{A. The Clock}

In this paper, we describe a modified version of the combinatorial clock auction [11] which reduces the complexity for both the bidders and auctioneers, making this mechanism feasible for a deployment in a network setting. Generally, auctions that provide feedback produce more efficient outcomes. In an iterative auction, as compared with say a single-shot sealed bid auction, bidders have an opportunity to explore the bid-space as their bids are either accepted or rejected during bidding rounds until there is no change in the winners or no new bids are submitted.

The clock auction operates in two stages: the iterative clock phase and the final assignment. The essence of the clock is that each unique item for sale has a price (a clock) associated with it. The price can only be adjusted (ticked) upwards in response to excess demand for that item. The cost of any package of items is equal to the sum of the individual prices for those items at that time, i.e. the auction yields anonymous linear prices.

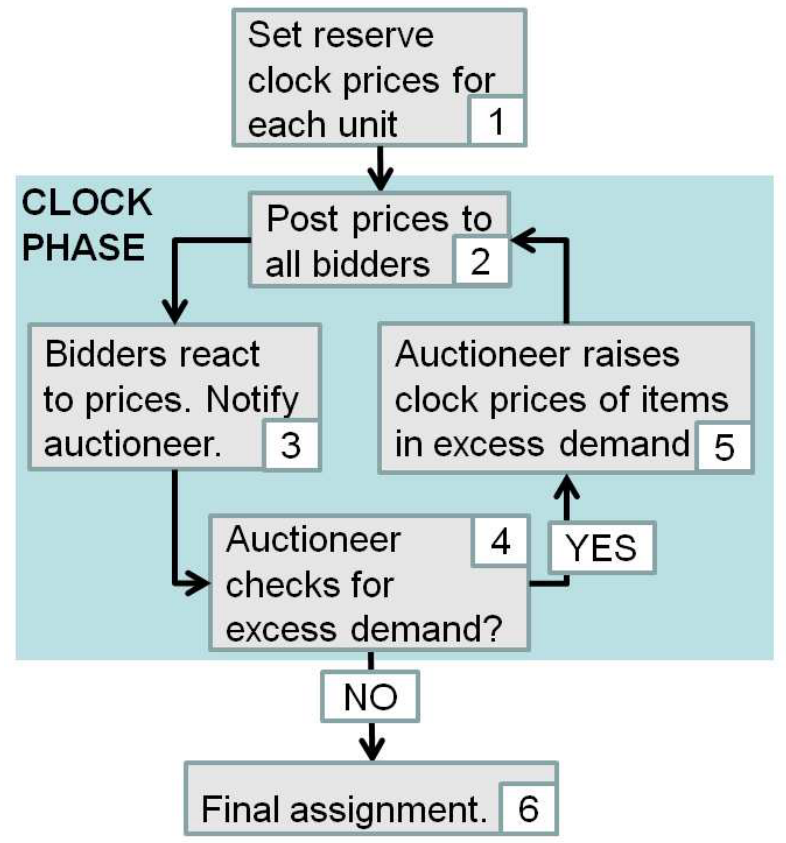

Fig. 3. The auction algorithm: clock phase and final assignment.

Briefly, the mechanism of the clock auction operates according to the following steps, as depicted in Figure 3:

1) Reserve: The auctioneer sets the initial clock price for each item. The initial price may reflect a seller's reserve price or it may reflect an impartial auctioneer's estimation of appropriate starting prices.

2) Post Prices: The auctioneer posts (broadcasts) the current clock prices for all items to all participants.

3) Bid: Bidders, i.e. buyers and sellers, submit bids to the auctioneer within a fixed period of time. Bid packages may be changed, parked or withdrawn.

4) Excess: The auctioneer determines if there is excess demand for any item. If so, it proceeds to step 5. If there is no excess demand for any item the auctioneer proceeds to step $\mathbf{6}$.

5) Tick: The auctioneer raises the clock price for items in excess demand and goes to step 2.

6) Assignment: The auctioneer assigns the items to the standing winning bidders.

The key characteristic of the clock auction is the relative simplicity of the price discovery phase, i.e. the clock phase. Firstly, its simplicity means that the burden on both the bidders and the auctioneer is low. The bidder simply has to sum the cost of any items it wants to package, ascertain that they are within its budget for that resource and make its bid. Depending on the flexibility of the bidding language, i.e. whether OR bids, XOR 
bids, etc. are allowed, the assessment of excess demand is relatively trivial for the auctioneer. The aim of the auctioneer during the clock phase is not to optimally allocate the packages, but rather to drive excess demand out of the system by raising prices without ending up with excess supply. Heuristic approaches can be used on a trial and error basis for this task.

Secondly, the posted price system that the clock auction uses lends itself to truthful price revelation and mitigates against strategic play. A bidder makes a bid knowing that the auction might terminate in that round, leaving it with a binding, winning bid. There is no bestand-final-offers round. Bidders cannot intimidate each other with large bid inmrements, they can only accept the auctioneers' posted prices. Nor can they see who else is bidding and what they are bidding. The only publicly shared information is the dissemination of the clock prices when a bidding round has ended.

Another key feature of the clock auction is that it is not a revenue-maximising auction, i.e. it does not operate solely for the benefit of the seller. The aim of this mechanism is to achieve all gains from trade and to be allocatively efficient.

\section{A Combinatorial Clock Auction for OFDMA-BASED ACCESS}

For an access technology trading scenario, the duration of the leases may be limited to a session duration, i.e. the lease length will be largely determined by the network characteristics (e.g. the stability/transience of network elements, the service being provided). In such a scenario, the auction mechanisms required are not so much for once-off initial-public-offering style trading, rather they will be for periodic repeated trading of rights from session to session. In this respect, the trading is more akin to a commodities market.

The traders in our system are the buyers, i.e. the subscriber stations, and the seller is the basestation. In our scenario, the SSs want to acquire fixed OFDMA-based backhaul capacity on the uplink and downlink to the basestation. OFDMA subcarriers are traded. Logically, the auctioneer should be a co-located entity in the SS, acting as a trusted-third-party. In [13], we have addressed a number of issues pertaining to the secure network implementation of general trading mechanisms.

We have modified the auction described in [11], in which (cognitive) human subjects participate as economically rational actors. Our initial network auction only allows AND bids covering the uplink and downlink, i.e. a bidder specifies the exact subcarriers in the downlink and uplink that it requires. For the purposes of computational simplicity in the event of an over-supply situation in the final assignment round, which would require the execution of an optimal allocation algorithm, we have restricted the bidding language to AND bids only, as indicated in Figure 4.

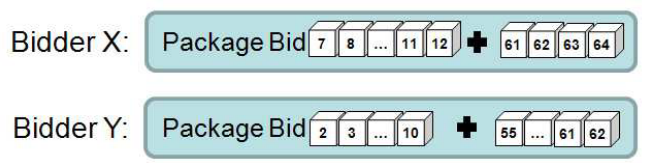

Fig. 4. Two sample AND packages: bidders indicating bins in uplink and downlink

Figure 5 illustrates a series of clock prices for OFDM subcarriers. In this instance, the initial (reserve) price is set equally for all items. As the rounds progress the clock prices for each subcarrier increase (or not) according to the bids submitted in that round. Eventually, the clock phase terminates. A problem for a time-limited/constrained network implementation centres on the non-deterministic nature of number of rounds required to remove excess demand. An aggressive clock adjustment policy ensures that excess is removed quickly, but it may cause much over supply which demands the execution of a burdensome winner determination allocation algorithm. Contrarily, a timid adjustment policy may find a price equilibrium, removing excess demand whilst not introducing excess supply - but at a cost of time.

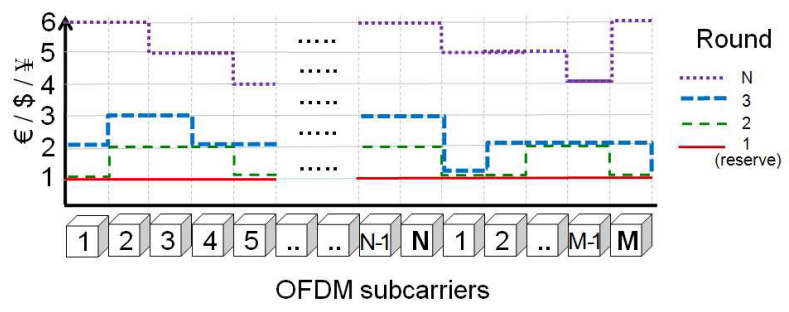

Fig. 5. Broadcast Information: Clock prices for items/OFMDA-bins 1to $\mathrm{N}$ (downlink) and 1 to $\mathrm{M}$ (uplink) over rounds 1 to $\mathrm{N}$.

In the network-based clock auction, the actions taken by the bidders and the auctioneer are automated and reactionary, i.e. the bids of each buyer are submitted and automatically adjusted with respect to a simple objective, i.e. bandwidth required as expressed in subcarriers, and simple constraint, i.e. a monetary budget.

The scope for cognition within this type of exclusive rights access paradigm manifests itself primarily in two places, the bidders and the auctioneer. The clock auction 
is not itself cognitive. Rather it is a mechanism that can be optimally exploited by cognitive participants. In order to properly replicate a real market in which there is repeated interaction, learning and strategising, cognition is required.

1) If one moves to an exclusive access paradigm, as against a QoS one, then the onus is on the bidders (buyers/sellers) to internalise the costs, benefits and risks of trading spectrum rights.

For a cognitive bidder, strategic decisions about what set of items (e.g. subcarriers) to bid for could be influenced by a number of factors; current price, historical session price if the bidder is in a repeat trading session, budget, quality of spectrum, intended service, etc.

2) From the clock auctioneer's point-of-view, the objective is to quickly terminate each auction in an efficient manner. In a time sensitive scenario, such as that involving the allocation of network access rights, a clock auction may fail if it cannot adjust its policies to take account of parameters that may effect the efficacy of its core mechanism. By avoiding, or minimizing, clock overshoots, and the consequent possibility of over-supply of items, the auctioneer can bring the auction to a conclusion with a simpler final assignment round. As with trader cognition, an auctioneer could use historical session data to calibrate the sizes of ticks for each item at each round.

\section{Conclusions}

Aside from the economic benefits of a clock auction, of more importance within the context of cognitive wireless networks is the space that this auction creates for unilateral and multilateral cognitive processing. The clock auction splits the processing load between all of the participants in the auction.

The simple messages that are exchanged between the auctioneer and the bidders abstract the, possibly heterogeneous, cognitive functionality of each individual entity which is free to use it own cognitive algorithms, localised information and experience to inform its decision-making and planning.

As we have discussed in this paper, the successful use of combinatorial clock auctions in a network-based setting is dependent on the injection of cognitive functionality into the mix.

\section{ACKNOWLEDGEMENT}

This material is based upon works supported by Science Foundation Ireland under Grant No. 03/CE3/I405.

\section{REFERENCES}

[1] Lehr, B., and Crowcroft, J., "Managing Shared Access to a Spectrum Commons", in Proceedings of IEEE DySPAN 2005, Balitmore, USA, Nov, 2005.

[2] T. Hazlett, The Spectrum-Allocation Debate: An Analysis, in IEEE Internet Computing, September/October 2006, pp 52-58.

[3] Kwerel, Evan R. and John R. Williams, "Free the Spectrum: Market-Based Spectrum Management," in Tom W. Bell and Solveig Singleton, eds., Regulators' Revenge: The Future of Telecommunications Deregulation. Washington, D.C.: Cato Institute, 1998, pp. 101-111.

[4] Kwerel, Evan R. and John R. Williams, "Moving toward a Market for Spectrum,” Regulation, 2 (1993), pp. 53-62.

[5] Kwerel, Evan R. and Alex D. Felker, "Using Auctions to Select FCC Licensees," Office of Plans and Policy Working Paper No. 16. Washington, D.C.: FCC, 1985.

[6] Second Report and Order, Order on Reconsideration, and Second Further Notice of Proposed Rulemaking (FCC 04167):Promoting Efficient Use of Spectrum Through Elimination of Barriers to the Development to Secondary Markets, Federal Communications Commission, September 2004.

[7] Cordeiro, C. M., Challapali, K., Birru, D. and Shankar,S., "IEEE 802.22: An Introduction to the First Wireless Standard based on Cognitive Radios", First IEEE International Symposium on New Frontiers in Dynamic Spectrum Access Networks (DySPAN), November 8 - 11, 2005, pp. 328 - 337.

[8] De Vany, A., "Implementing a Market-Based Spectrum Policy", Journal of Law \& Economics, 41 (October 1998), pp. 627-646.

[9] De Vany, Arthur S., Ross D. Eckert, Charles J. Meyers, Donald J. O'Hara, and Richard C. Scott, "A Property System for Market Allocation of the Electromagnetic Spectrum: A LegalEconomic-Engineering Study," Stanford Law Review, 21 (June 1969), pp. 1499- 1561.

[10] Mitola, J., III and Maguire, G.Q., Jr., "Cognitive radio: making soft- ware radios more personal", IEEE Personal Communications [see also IEEE Wireless Communications], August 1999, 6(4),13 - 18.

[11] Porter, D., Rassenti, S., Roopnarine, A., and Smith V., "Combinatorial auction design", Proceedings of the National Academy of Sciences of the United States of America, September 2003, 100(19), pp: 11153 - 11157.

[12] Sandholm, T., "Expressive Commerce and Its Application to Sourcing: How We Conducted \$35 Billion of Generalized Combinatorial Auctions", AI Magazine, 28(3), 45-58, Fall 2007.

[13] Argyroudis, P., Forde, T., Doyle, L. and O'Mahony, D. "A Policy-driven Trading Framework for Market-based Spectrum Assignment", in Proceedings of 8th IEEE International Workshop on Policies for Distributed Systems and Networks (Policy'07), 13-15 June 2007, Bologna, Italy.

[14] Cramton,P., Shoham, Y, and Steinberg, R., (editors), "Combinatorial Auctions", MIT Press, 2006. ISBN:0262033429.

[15] Federal Communications Commission, Unlicensed operation in the TV broadcast bands. ET Docket No. 04- 113, May 2004. 